\title{
Studying mass transport dynamics in polymer electrolyte membrane fuel cells using concentration-alternating frequency response analysis
}

\author{
A. Sorrentino ${ }^{a}$, T. Vidakovic-Koch ${ }^{\mathrm{a},},{ }^{*}$, K. Sundmacher ${ }^{\mathrm{a}, \mathrm{b}}$ \\ ${ }^{a}$ Max Planck Institute for Dynamics of Complex Technical Systems, Sandtorstraße 1, D-39106 \\ Magdeburg, Germany \\ ${ }^{b}$ Otto-von-Guericke University Magdeburg, Process Systems Engineering, Universitätsplatz 2, \\ D-39106 Magdeburg, Germany \\ *Corresponding author: vidakovic@mpi-magdeburg.mpg.de
}

\begin{abstract}
Key words: polymer electrolyte membrane fuel cell dynamics; mass transport; electrochemical impedance spectroscopy; frequency response analysis; system identification; periodic concentration input
\end{abstract}

\begin{abstract}
In the present contribution an experimental validation and a measurement routine of a new electrochemical method to study dynamics of electrochemical processes, the so-called concentration alternating frequency response analysis (CFRA) is presented. In CFRA the electrical response of the cell (current or potential) to periodic perturbation of specific reactant feeds is studied by means of linear system analysis. For the example of a polymer electrolyte fuel cell (PEMFC) we show that cFRA, in contrast to classical electrochemical impedance spectroscopy (EIS), detects selectively the effect of the dynamics of mass transport of reactants and products inside the different layers of the PEMFC. Moreover, cFRA can be used to diagnose the humidification state of the fuel cell cathode. Finally,
\end{abstract}


procedures to improve the diagnostic skills of the proposed cFRA technique are discussed and directions of future work are recommended.

\section{Introduction}

Electrochemical impedance spectroscopy (EIS) is the most often used experimental technique to unravel the complex dynamics of polymer exchange fuel cells (PEMFC) [1, 2]. It is based on the excitation of the cell at a certain steady state through a periodic electrical input, current or cell potential, and the detection of the resulting electric response at different frequencies. The input/output correlation is inspected by means of linear frequency response analysis (FRA) through the formulation of a transfer function in the frequency domain. Electric stimulations are able to identify almost all dynamic processes with different time constants occurring in the cell, i.e. mass transport of reagents and products, as well as chemical and electrochemical reactions at the electrode interface. However, some of the phenomena involved are strongly coupled in the EIS spectra, thus they do not allow the deconvolution of all subprocesses appearing in the fuel cell. Therefore, the development of novel in-situ diagnostic tools is required in order to analyse selectively individual phenomena and add additional insight to the comprehension of the impact of PEMFC transients on the performance. During the last years, several groups have proposed FRA experimental techniques based on nonelectrical inputs and/or outputs to study the dynamics of electrochemical systems [3-6]. Recently, the use of the backpressure as input variable was suggested for studying the transport phenomena in the PEMFC $[7,8]$. Most notably, Engebretsen et al. have proposed a transfer function involving the cathode backpressure perturbations as input and the cell potential as output [8]. A quantitative correlation between amplitude and phase angle with the water imbalances was found.

Recently, our group has proposed a new FRA technique named concentration alternating frequency response analysis (cFRA) which is based on transfer functions depending on partial pressure 
perturbations of specific reactants [9]. Application of cFRA for studying the PEMFC dynamics was investigated theoretically in our previous publication, where we revealed the possibility of identifying selectively different transport phenomena by the new technique [9]. In the present communication, we report an experimental setup able to perform cFRA experiments under voltastatic and galvanostatic control. The analysis of the measured transfer functions unravels various aspects related to gas and water transport in the different layers of the cell. A comparison with the theoretical results is also given. Moreover, it was found that the cFRA method can be used to diagnose the humidification state of the cathode. Finally, future improvements of the proposed technique are discussed.

\section{Experimental}

All experimental tests were performed using a single PEMFC having an active area of $25 \mathrm{~cm}^{2}$ and flow fields with parallel channel configuration. The membrane electrode assembly (MEA) was prepared in house following the same procedure as described in Kadyk et al. [10], using a Nafion 117 membrane and a catalyst ink consisting of platinum black (Alpha Aesar). The platinum loading in both catalyst layers (CL) was $1 \mathrm{mg} / \mathrm{cm}^{2}$. Toray paper TGP-H-060 was used as gas diffusion layer (GDL). The temperature of the cell was fixed at $70{ }^{\circ} \mathrm{C}$ and was controlled by a heating box equipped with a PID controller. An Autolab potentiostat PGSTAT302 was used to control and monitor the cell potential and current during the experiments.

A feed of pure hydrogen with a flow rate fixed at $800 \mathrm{ml} / \mathrm{min}$ was supplied to the anode, while the cathode was fed with $250 \mathrm{ml} / \mathrm{min}$ of nitrogen and oxygen. Dew point temperatures were $60^{\circ} \mathrm{C}$ and $40{ }^{\circ} \mathrm{C}$ for the cathode and anode feed stream, respectively. These conditions of wet cathode/almost dry anode induced a significant backwards permeation of water in the membrane.

A scheme of the cathode conditioning section of the setup is displayed in figure 1 . Streams of nitrogen (1) and oxygen (2) were supplied to the cathode of the fuel cell (11) in the desired quantity and proportions. The cathode feed was humidified using a bubble humidifier (4) where the 
temperature of water was controlled by the use of a thermostat equipped with a circulation loop (6).

The dew point temperature and the relative humidity were measured by a dew point sensor (7). After the bubbler, an additional stream of oxygen (3) was periodically imposed to the cathode main stream through the use of a control valve (5) which was able to switch the stream to the cathode inlet or to the outlet. By regulating the switching velocity of the valve, it was possible to generate a periodic inlet signal of oxygen partial pressure at different frequencies. The proportion of the additional oxygen stream (3) was kept lower than $10 \%$ of the cathode main flow rate to assure oxygen perturbation small enough to have a linear electric response of the fuel cell (11). The variation of oxygen pressure was measured at the cell inlet through an optical fiber sensor (OXR430UHS Pyro Science) (9). The sensor has a sampling frequency of $6.6 \mathrm{~Hz}$ that, according to the NyquistShannon theorem, allowed detection of a maximal frequency of $3.3 \mathrm{~Hz}$ in the spectra. The variation of the total pressure consequent to the added flow was measured, too. A fluctuation of $30 \mathrm{~Pa}$ from the average value of the inlet pressure was detected. This value is negligible compared to the registered variation of the partial pressure of oxygen of $1000 \mathrm{~Pa}$ at low frequencies input. Therefore, the electric response of the cell was due to the change of partial pressure of specific components in the main stream unlike the study by Engrebtsen et al [8].

The electric response of the cell to the feed variations depends on the electric control applied on it. A periodic current or periodic cell potential is obtained if the cell is kept at constant cell potential or current, respectively.

\section{Theoretical background and data analysis}

The correlation between pressure input/electric output at different frequencies is analysed by means of linear FRA by determining a proper transfer function. The cFRA transfer functions for the perturbation of a specific component $\alpha$ under voltastatic or galvanostatic control read as follows:

$\zeta_{\alpha}^{V}=\frac{\Delta I(\omega)}{\Delta P_{\alpha}(\omega)}$ 


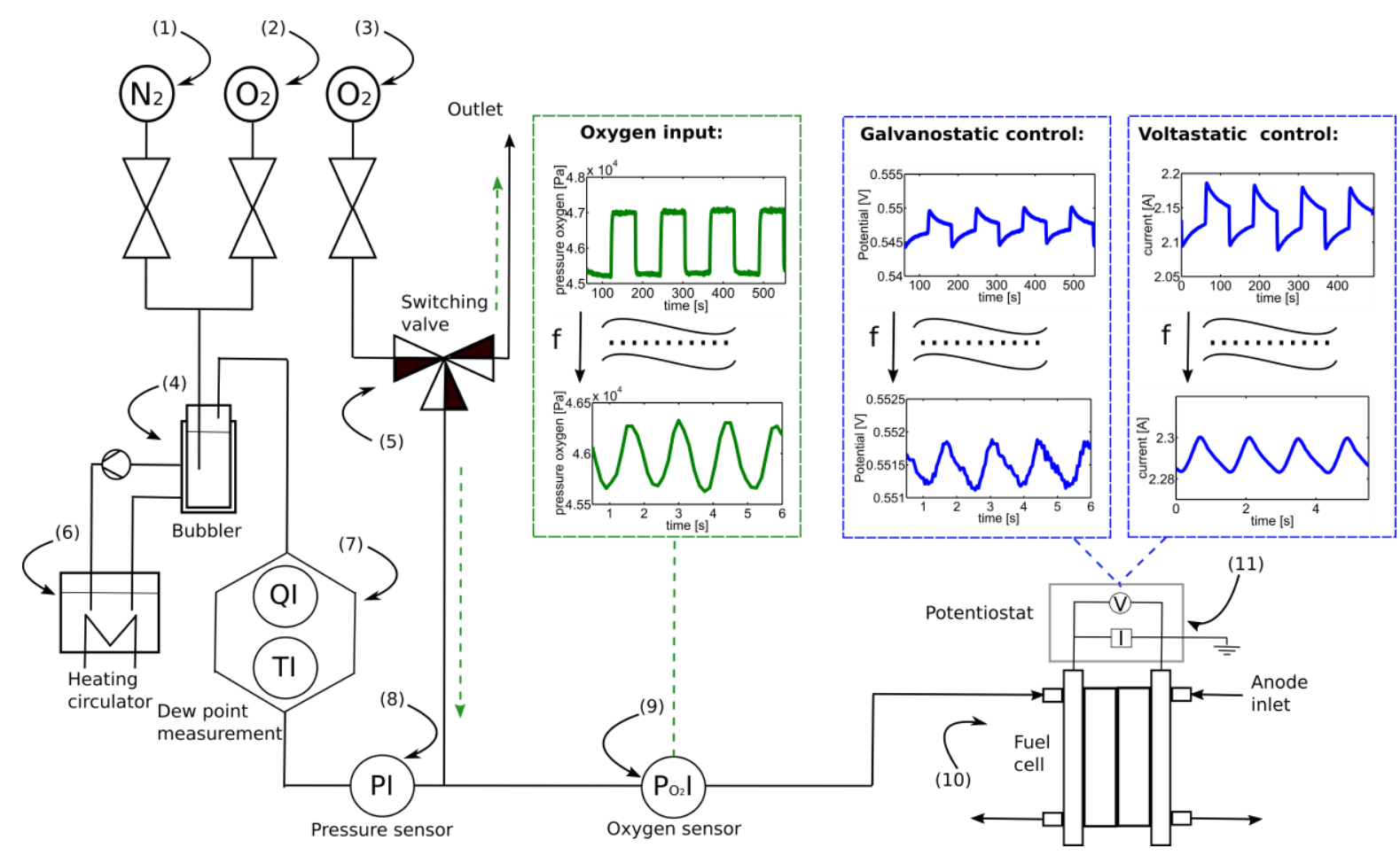

Figure 1: Schematic diagram of the cathode feed conditioning section of the experimental setup.

Periodic input signal (oxygen pressure) and electric output signals (cell potential or current) at two different frequencies are shown in the insets.

However, with respect to the data analysis one has to keep in mind that in the present experimental setup, the periodic addition of the small oxygen flow after the bubble humidifier causes not only a variation of the oxygen pressure, but a variation of the water vapour pressure as well. Basically, an increment of the oxygen partial pressure excites a complementary decrement of the partial pressure of water and vice versa, resulting in a simultaneous periodic stimulation where the two concentration inputs $\left(\mathrm{O} 2\right.$ and $\left.\mathrm{H}_{2} \mathrm{O}\right)$ are in antiphase. This indicates further that the experimentally measured CFRA transfer functions are mixed transfer functions containing contributions of the transfer functions of both $\mathrm{O}_{2}$ and $\mathrm{H}_{2} \mathrm{O}$. By registering the variation of oxygen pressure and the electric 
outputs at each frequency $f$, according to the facts exposed, instead of the theoretical cFRA (eqs.1-

2), the following mixed oxygen cFRA transfer function, $\zeta_{O_{2}}^{V}{ }^{\prime}$ and $\zeta_{O_{2}}{ }^{\prime}{ }^{\prime}$, were measured:

$\zeta_{O_{2}}^{V}{ }^{\prime}(\omega)=\frac{\Delta I(\omega)}{\Delta P_{O_{2}}(\omega)}=\zeta_{O_{2}}^{V}(\omega)+\beta \zeta_{H_{2} O}^{V}(\omega)$

$\zeta_{O_{2}}^{I}{ }^{\prime}(\omega)=\frac{\Delta V(\omega)}{\Delta P_{O_{2}}(\omega)}=\zeta_{O_{2}}^{I}(\omega)+\beta \zeta_{H_{2} O}^{I}(\omega)$

The variable $\beta=\frac{\Delta P_{\mathrm{H}_{2} \mathrm{O}}}{\Delta P_{\mathrm{O}_{2}}}$ quantifies the fraction of the water cFRA transfer function overlapping with the experimental oxygen cFRA transfer function. The value of $\beta$ depends on the specifications of the main flow and it is assumed to be constant over all frequencies. Since $\Delta P_{\mathrm{H}_{2} \mathrm{O}}$ and $\Delta P_{\mathrm{O}_{2}}$ have opposite signs, the contribution of the water cFRA transfer function is subtractive.

For analysing input and output periodic signals, at least 5 cycles at each frequency were registered. Then, the Fourier transform of the signal was determined. As shown in the insets in figure 1, the signals had a sinusoidal shape at the highest frequencies. They turned into a periodic square wave at lower frequencies. For this reason, the frequency spectra derived from the Fourier transform at lower frequencies contained different harmonic signals in addition to the main frequency. For assuring that the cFRA spectra were rectified from noise effects, only the harmonics of the most important frequency were registered.

\section{Results and discussion}

CFRA experiments were performed in order to verify the results of our previous theoretical work [9]. All the theoretical calculations are based on simulations of a PEMFC dynamic model which basically includes gas transport in the channel and in the GDL, transport of water in the Nafion membrane, and the double layer charging/discharging at catalyst surface. The catalyst layer was considered as an interface, while the effect of liquid water formation was neglected overall in the cell. To limit the impact of the latter assumption on the experiments and validate the model predictions, low currents 
and not fully humidified feed were used in experiments, as explained in more detail in the previous section. Specifically, experimental voltastatic and galvanostatic oxygen cFRA spectra were recorded at three different steady state current densities and steady state cell potentials respectively (figure 2: a-d). As can be seen, experimental cFRA spectra were almost independent on the cell steady state current/potential at the highest frequencies applied $(f \approx 1 \mathrm{~Hz})$. On the contrary, they showed very pronounced dependence on the operating conditions in the lower frequency range. The negligible difference between the cFRA spectra at different cell potentials/cell currents at high frequencies can be explained by the time delay between the input signal perturbation (oxygen concentration change) and the system response in terms of current and overpotential changes, as it was also discussed in our theoretical study [9]. Since the charge transfer kinetic effects are usually observable at high frequencies $[1,2]$, this suggests that the cFRA technique is not very sensitive with respect to the charge transfer resistances at the catalyst interface and that it is not capable of detecting the related dynamics. On the other hand, the cFRA method shows high sensitivity for mass transport dynamics and can be regarded as a very suitable method to study selectively the impact of different mass transport phenomena on the PEM fuel cell performance.
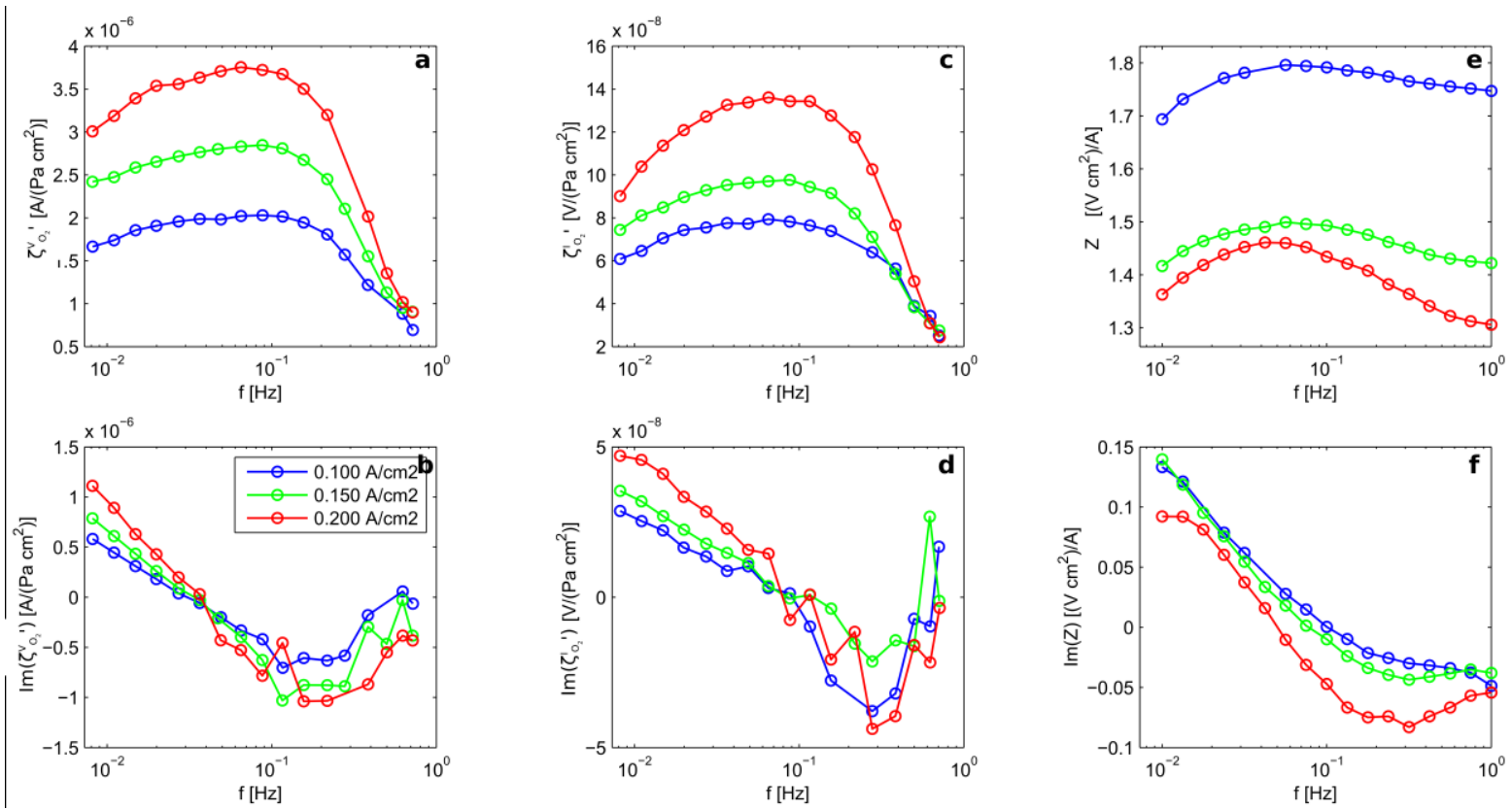
Figure 2: Magnitude (top) and imaginary part (bottom) spectra of voltastatic cFRA (a-b),

galvanostatic cFRA (c-d) $\beta=0.45$ and conventional EIS (e-f).

For comparison purposes, traditional galvanostatic EIS spectra collected at the same steady state conditions as the CFRA are shown in figures 2e-f. EIS magnitudes in general decrease with an increment of overpotential [1, 2]. Unlike cFRA spectra, EIS magnitude spectra (figure: 2e) are strongly dependent on the cell current (cell potential) in the whole frequency range. Basically, two distinct transients could be detected through the inflection points of the EIS magnitude spectra in the considered frequency range $\left(10^{-2}-1 \mathrm{~Hz}\right)$. The characteristic frequencies related to these phenomena can be identified from the turning points of the imaginary part of the impedance (figure: $2 \mathrm{f}$ ). The dynamics of gas transport in the cathode, and concentration polarization losses due to liquid water transport in the catalyst layer $(\mathrm{CL})$ are observable in the frequency range $0.056-0.8 \mathrm{~Hz}$ [11-14]. The time constant of the gas replacement in the PEMFC flow field was estimated from the minimum point observed at the higher frequencies of the imaginary part plot of the different transfer functions (figure $2 \mathrm{~b}-\mathrm{f}$ ). The estimated time constant is in the range between 0.5 and $0.8 \mathrm{~s}$ At lower frequencies, the decrement of the magnitude and imaginary part of the impedance approaching $0.01 \mathrm{~Hz}$ can be attributed to the hydration of the Nafion membrane by backwards diffusion of water formed at the cathode [15-16]. Both transport phenomena can be observed in the cFRA spectra through similar patterns. 

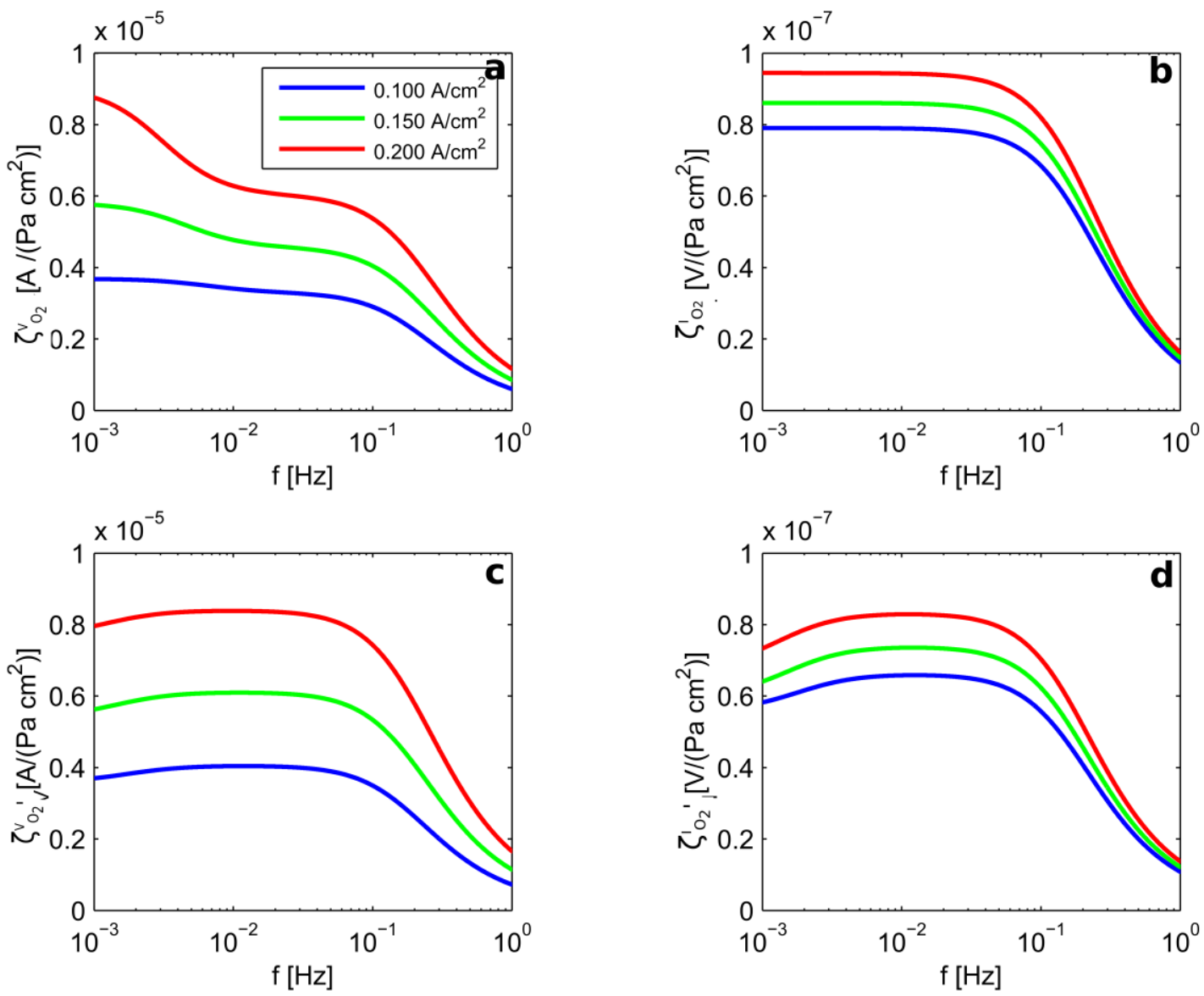

Figure 3: Simulated magnitude plots of the oxygen cFRA transfer function: a) pure voltastatic oxygen cFRA transfer function (eq.2), b) pure galvanostatic oxygen cFRA transfer function (eq.1), c) coupled voltastatic cFRA transfer function (eq.4), d) coupled galvanostatic cFRA transfer function (eq.3).

The magnitudes of oxygen cFRA transfer functions (eqs.1-2) (figures 3a-b) are calculated assuming the same conditions as in the experiments with the help of a mathematical model developed in our previous publication [9]. As can be seen, at frequencies higher than $0.1 \mathrm{~Hz}$ the experimental oxygen cFRA magnitudes agree qualitatively well with the theoretically calculated cFRAs. However, at lower frequencies there are discrepancies between theoretical and experimental oxygen cFRA spectra, which can be mainly assigned to contributions from the simultaneous perturbation of the water content of the gas feed (eqs.3-4). According to the theory [9] the magnitudes of the voltastatic cFRAs spectra (figure 3a) at lower frequencies should increase. This is a consequence of the electric current increase caused by lowering of the Ohmic resistance due to membrane hydration (figure 3a). 
Therefore, the observed decrement in the experimental voltastatic spectra (figure $2 a-b$ ) are presumably due to the antiphase contributions of the water pressure which diminish this beneficial effect. To support this hypothesis, magnitudes of voltastatic oxygen cFRA accounting for the simultaneous water perturbation (eqs.3-4) were calculated (see figure 3c). As can be seen, the calculated spectra reflect the trend of the experimental ones. Furthermore, as already discussed in our theoretical study [9], under galvanostatic control, there is a constant water production in the catalyst layer, which implies an unchanged hydration level of the membrane and consequently a constant value of the cFRA magnitude in the frequency range below $0.1 \mathrm{~Hz}$ (figure: $3 \mathrm{~b}$ ). However, in the experimental spectra the subtractive contribution of the antiphase water perturbation, changes the water content in the membrane, and a decrement in the spectra is observed in the experiment (figure $2 \mathrm{a}-\mathrm{b}$ ) and predicted by the simulations under same conditions (figure $3 \mathrm{~d}$ ). The influence of the simultaneous water perturbation is further demonstrated experimentally, as can be seen in figure 4 .

Voltastatic cFRA and EIS spectra were recorded experimentally at a steady state electric current density $100 \mathrm{~mA} \mathrm{~cm}^{-2}$ using two different experimental conditions, namely a dry anode/wet cathode configuration, and a dry anode/dry cathode configuration. In the first case, the calculated fraction of the water CFRA transfer function is $\beta=0.45$, while in the second case a lower value of $\beta=0.15$ presents a lower influence of the overlapping water perturbation. Unlike EIS spectra (figure: 4a) which cannot differentiate between two cathode states (in both cases, strong membrane hydration results in lowering the EIS magnitude at low frequencies), the voltastatic CFRA method showed a qualitative difference of the magnitude spectra at low frequencies. Namely, for the voltastatic cFRA an increment was observed with the dry anode/dry cathode configuration, while a decrement of the magnitude was obtained with the dry anode/wet cathode configuration (figure: 4b). This pattern suggests that at lower $\beta$ values the contribution of the water perturbation becomes smaller, thus the apparent oxygen cFRA gets closer to theoretically expected cFRA. This confirms the hypothesis of the 
beneficial effect of the water produced under voltastatic condition. These results show that the cFRA method can be used for diagnosis of the humidification state (wet or dry) of a PEM fuel cell cathode.
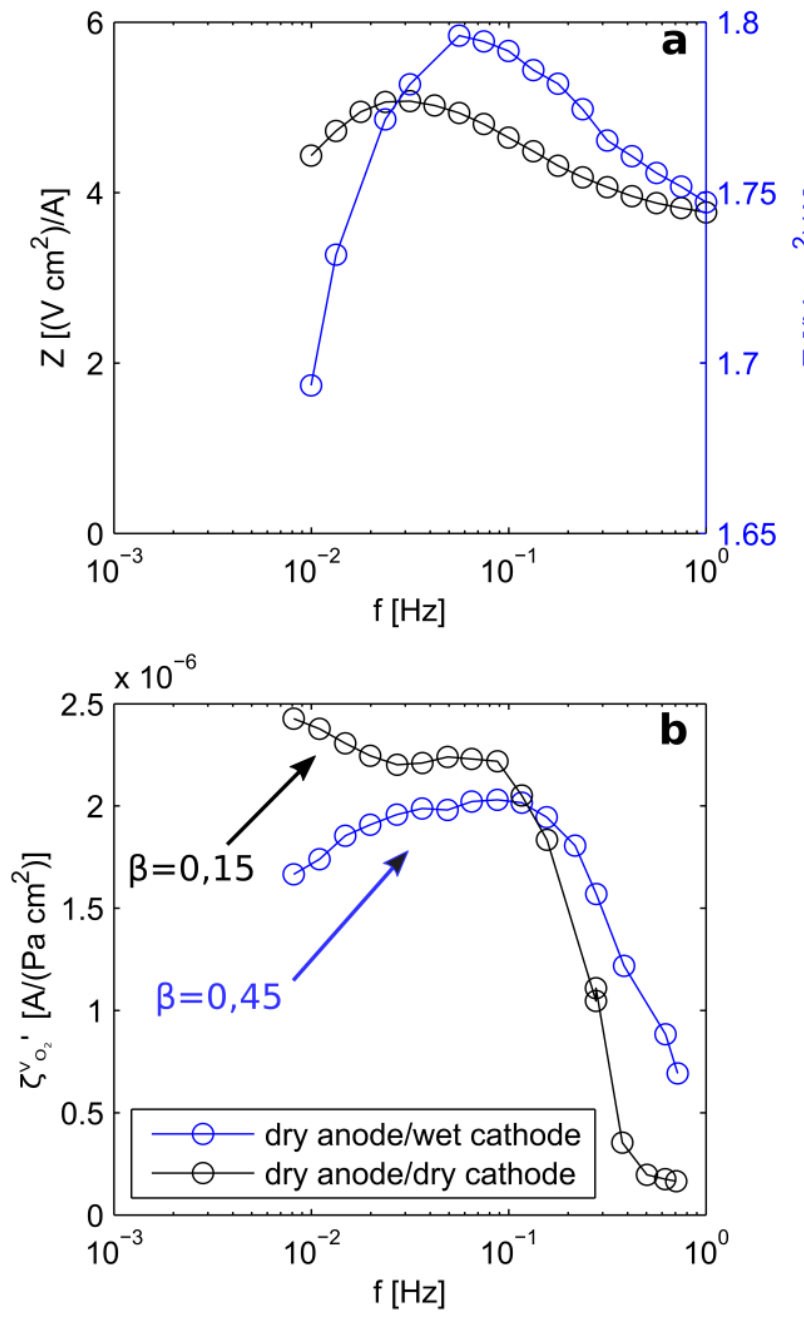

Figure 4: Magnitude spectra of traditional EIS (a) and potentiostatic CFRA (b) at a steady state current of $100 \mathrm{~mA} \mathrm{~cm}^{-2}$ for dry anode/wet cathode (dew point temperature anode and cathode feed respectively $40^{\circ} \mathrm{C}$ and $60^{\circ} \mathrm{C}$ ) and dry anode/dry cathode configurations (dew point temperature of anode and cathode feed at $\left.30^{\circ} \mathrm{C}\right)$.

\section{Strategy to decouple concentration inputs contribution}

As discussed above, the simultaneous excitation of the system by water and oxygen partial pressures does not allow to measure the pure transfer functions $\zeta_{\mathrm{O}_{2}}^{V(I)}$ and $\zeta_{\mathrm{H}_{2} \mathrm{O}}^{V(I)}$, but only coupled ones. Therefore, the possibility of selectively detecting the dynamics related to the gas and water transport in a PEM fuel cell, as predicted by our theoretical study, cannot be fully verified through the here described operating procedures [9]. One approach to decouple the contributions of the two 
concentration inputs and to obtain the pure transfer functions is to measure two linearly independent sets of cFRA data for the system. Considering the cell at certain steady state condition and fixed specifications of the inlet flow rate, two cFRA experiments can be performed using different flow variations characterized by two different oxygen-water ratios, $\beta^{\prime}$ and $\beta^{\prime \prime}$. Thereby, two coupled transfer functions, which are linear combinations of the pure ones, are obtained:

$$
\begin{aligned}
& \zeta_{O_{2}}^{V(I) \prime}(\omega)=\frac{\Delta I^{\prime}(\omega)}{\Delta P_{O_{2}}{ }^{\prime}(\omega)}=\zeta_{O_{2}}^{V(I)}(\omega)+\beta^{\prime} \zeta_{H_{2} O}^{V(I)}(\omega) \\
& \zeta_{O_{2}}^{V(I) \prime \prime}(\omega)=\frac{\Delta I^{\prime \prime}(\omega)}{\Delta P_{O_{2}}{ }^{\prime \prime}(\omega)}=\zeta_{O_{2}}^{V(I)}(\omega)+\beta^{\prime \prime} \zeta_{H_{2} O}^{V(I)}(\omega)
\end{aligned}
$$

The mathematical system constituted by equations (5) and (6) can be solved for each frequency and thus the pure oxygen and water transfer function are extracted:

$$
\begin{aligned}
& \zeta_{O_{2}}^{V(I)}(\omega)=\left(\frac{\beta^{\prime \prime}}{\beta^{\prime \prime}-\beta^{\prime}}\right) \zeta_{O_{2}}^{V(I) \prime}(\omega)+\left(-\frac{\beta^{\prime \prime}}{\beta^{\prime \prime}-\beta^{\prime}}\right) \zeta_{O_{2}}^{V(I) \prime \prime}(\omega) \\
& \zeta_{O_{2}}^{V(I)}(\omega)=\left(-\frac{1}{\beta^{\prime \prime}-\beta^{\prime}}\right) \zeta_{O_{2}}^{V(I) \prime}(\omega)+\left(\frac{1}{\beta^{\prime \prime}-\beta^{\prime}}\right) \zeta_{O_{2}}^{V(I) \prime \prime}(\omega)
\end{aligned}
$$

The main requirement to apply this calculation scheme to experimental data is the linear independency of equations (5) and (6).

In our lab, an experimental setup able to vary the flow rate with different values of $\beta$ has been developed. An extended paper showing the spectra of the decoupled cFRA transfer functions will be published in the future.

\section{Conclusion}

First experiments with the cFRA technique were performed with a laboratory scale PEM fuel cell. Unlike conventional EIS, cFRA spectra are not affected by charge transfer processes at high frequencies and can detect selectively the dynamics related to the transport of the reagents in the electrodes and transport of water inside of the Nafion membrane. It was shown that the cFRA method can be used to diagnose problems related to the humidification state of the cathode. The 
presented experimental setup enables to determine cFRA transfer functions which contains coupled contributions of oxygen and water variations to the output. For this reason, the ability to study selectively specific transport dynamics which was predicted by theoretical studies has not been observed. A strategy to decouple the contributions of the concentration inputs has been presented to overcome this problem.

\section{References}

[1] X. Yuan, H. Wang, J. C. Sun, and J. Zhang. AC impedance technique in PEM fuel cell diagnosis - a review. International Journal of Hydrogen Energy, 32(7):4365 - 4380, 2007.

[2] S. M. Rezaei Niya and M. Hoorfar. Study of proton exchange membrane fuel cells using electrochemical impedance spectroscopy technique - a review. Journal of Power Sources, 240(8):281-293, 2013.

[3] C. Gabrielli and B. Tribollet. A transfer function approach for a generalized electrochemical impedance spectroscopy. Journal of the Electrochemical Society, 141(5):1147 - 1146, 1994.

[4] D. D. Macdonald, E. Sikora, and G. Engelhardt. Characterizing electrochemical systems in the frequency domain. Electrochimica Acta, 43(1):87 - 107, 1998.

[5] J. R. Collet-Lacoste. The electrochemical impedance spectroscopy and associated transfer functions: non-equilibrium thermodynamics consideration. Electrochimica Acta, 49(5):4967 4977, 2004.

[6] M. Orazem and B. Tribollet. Electrochemical Impedance Spectroscopy. Wiley, 2008.

[7] A. M. Niroumand, W. Merida, M. Eikerling, and M. Safi. Pressure voltage oscillations as diagnostic tool for PEFC cathode. Electrochemistry Communications, 12(1):122 - 124, 2010. 
[8] E. Engebretsen, T.J. Mason, P.R. Shearing, G. Hinds, and D.J.L. Brett. Electrochemical pressure impedance spectroscopy applied to the study of polymer electrolyte fuel cells. Electrochemistry Communications, 75:60-63, 2016.

[9] A. Sorrentino, T. Vidaković-Koch, R. Hanke-Rauschenbach, and K. Sundmacher. Concentration frequency response analysis: A new method for studying polymer electrolyte membrane fuel cell. Electrochimica Acta, 243:53 - 64, 2017.

[10] T. Kadyk, R. Hanke-Rauschenbach, and K. Sundmacher. Nonlinear frequency response analysis of PEM fuel cells for diagnosis of dehydration, flooding and co-poisoning. Journal of Electroanalytical Chemistry, 630:19-27, 2009.

[11] T. D. Springer, T. A. Zawodzinski, M. S. Wilson, and S. Gottesfeld. Characterization of polymer electrolyte fuel cells using ac impedance spectroscopy. Journal of the Electrochemical Society, 143(2):587 - 599, 1996.

[12] M. Ciureanu and R. Roberge. Electrochemical impedance study of PEM fuel cells: Experimental diagnostics and modeling of air cathodes. The Journal of Physical Chemistry B, 105(17):35313539, 2001.

[13] I.A. Schneider, M.H. Bayer, A. Wokaun, and G.G. Scherer. Impedance response of the proton exchange membrane in polymer electrolyte fuel cells. Journal of the Electrochemical Society, 155(8):B783-B792, 2008.

[14] D. Gerteisen, T. Heilmann, and C. Ziegler. Modeling the phenomena of dehydration and flooding of a polymer electrolyte membrane fuel cell. Journal of Power Sources, 187(1):165 - 181, 2009.

[15] N. Holmström, K. Wiezell, and G. Lindbergh. Studying low humidity effects in PEMFCs using EIS: I. experimental. Journal of the Electrochemical Society, 159(8):F369-F378, 2012.

[16] K. Wiezell, N. Holmström, and G. Lindbergh. Studying low-humidity effects in PEMFCs using EIS, II. modeling. Journal of the Electrochemical Society, 159(8):F379-F392, 2012. 